\title{
Crowdsourced Ethics with Personalized Story Matching
}

Henry Lieberman

Karthik Dinakar

Birago Jones

MIT Media Lab

20 Ames St.

Cambridge, MA 02139 USA

\{lieber, kdinakar, birago\}

@media.mit.edu

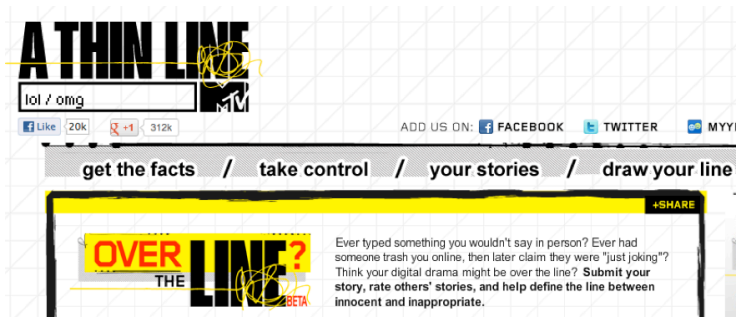

Figure 1. Intro to MTV's "Over the Line"

Copyright is held by the author/owner(s).

CHI 2013 Extended Abstracts, April 27 \{May 2, 2013, Paris,France.

ACM 978-1-4503-1952-2/13/04.

\begin{abstract}
Cyberbullying is a widespread and growing social

problem, threatening the viability of social networks for youth. We believe that one of the best ways to combat

this problem is to use these incidents as "teaching moments", encouraging teens to reflect on their behavior and choices. Sites that offer community discussions around the ethical aspects of social situations can help teens feel less alone in their plight, and provide useful advice and emotional support. But the success of these "crowdsourced ethics" sites depends critically on the user feeling like discussions are relevant to their own personal experience.

We have augmented the crowdsourced ethics site "Over The Line", offered by MTV Networks, with a personalized story matcher that classifies stories according to dynamically discovered high-level themes like "sending nude pictures online" or "feeling pressure in relationships". The matcher uses a mixed-initiative LDA machine learning technique [2], and a commonsense knowledge base specialized to the bullying problem. The site is currently public, and attracts an audience of thousands of users daily.
\end{abstract}




\section{Author Keywords}

Cyberbullying; Crowdsourcing; Commonsense reasoning; Personalization; Machine Learning;

\section{ACM Classification Keywords}

H.5.3 Collaborative Computing

\section{The Cyberbullying problem}

Cyberbullying, harassment of youth by their peers on online networks, is a growing social problem [1], [10]. Studies show that almost half of young people experience it at some point [8], and extreme cases can lead to tragedies such as suicides.

Cyberbullying presents the same kind of threat to participation by youth on social networks, that spam did to e-mail in the early days of the Internet. If we allow it to get too prevalent, it will destroy the viability of social networks as a communication medium for youth. Just as spam filters provided a technical solution that, while they didn't eliminate it, kept the problem under control, we need a new generation of technical solutions adapted to this particular problem.

\section{Reflective Interfaces}

We would like to avoid heavy-handed approaches, such as censoring a post or banning the perpetrator. Our general approach to interface design is called Reflective Interfaces [5]. Reflective Interfaces are designed to encourage the user to think about why they made the choices they did; what the consequences are for themselves and others, and their options for future action. This is in contrast to conventional interfaces, which focus on just the immediate content of the interface choice or the communication taking place.
Reflective Interface design borrows its framework from principles espoused by Donald Schön [12]. Schön talked about the need for successive stages of action, then reflection, which in turn can have influence on future action.

\section{Crowdsourced ethics: MTV's "A Thin Line" and "Over the Line" sites}

Many sites offer anti-bullying advice, such as the portal stopbullying.gov [14]. Effectiveness is limited, though, as advice tends to be vague (e.g. "Tell a trusted adult"), unconnected to a child's personal situation, and delivered far from the times and places when bullying actually occurs.

We chose to work with MTV Network's "A Thin Line" because it adopts a unique crowdsourced ethics approach. It encourages users to describe potentially problematic situations and engage in conversations about whether a situation is or is not "over the line". Even the "Thin Line" title recognizes that it shouldn't try to preach a single correct answer. It encourages the user to reflect on how their particular experience relates to the experience of others. MTV reports that the average user spends $9 x$ as long on the Over the Line? site than on portions of their site that merely present passive information [9].

Perhaps its greatest effect is to help the user realize that they are not alone, that their plight is shared by others, and that they can reach out to others for help. The most extreme negative reactions to bullying situations such as depression and even suicide, tend to occur only when a person feels like they're totally isolated in their predicament, and that there is no way out. 
The top level of the "Thin Line" site (Figure 1) acts as a portal, offering education on bullying topics, help lines, sign up for anti-bullying campaigns, and a blog. The "Your Stories" link leads to the "Over the Line" page, which is concerned with personal stories contributed by users. Users can simply read stories contributed by others, and they can also rate them "over", "on", or "under" the line of acceptability. Users can also see the results of how others rated a particular story.

\section{http://www.athinline.org/overtheline/}

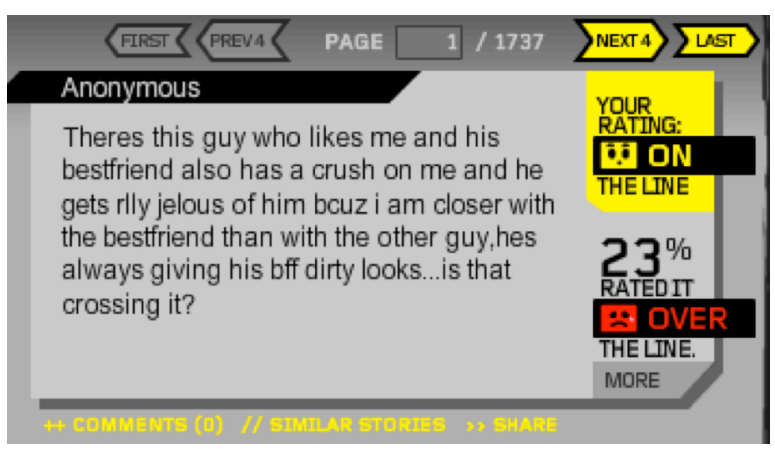

Figure 2. A story rated by the community

\section{Personalization for crowdsourced ethics by understanding high-level themes}

But the stories appear on the site with no particular topic organization. You can order them by recency, popularity, rating, or a (hand-selected) category "outrageous". But what's likely to make the stories most effective is to be able to find the ones that are most personally relevant for you.
Personalization for an application such as this is challenging. We can't rely on many traditional methods, such as keyword spotting, such as is typically used in spam filters, conventional topic modeling, and other information retrieval techniques. Just getting a superficial match to the particulars of the story is generally not helpful (even though a story mentions that it took place at a basketball game, other stories about basketball are probably irrelevant).

Our goal is, instead, to identify high-level themes of the story that are likely to be relevant to the topics and shared with other stories. This isn't the place to go into details of the story matching algorithm; for that see the references [3], [4]. The contribution of this paper is to present the user interface for the deployed public site.

Briefly, though, we employ a mixed-initiative variant of the machine learning technique Latent Dirichlet Allocation (LDA) [2]. LDA models a document as being produced from a set of underlying topics by a probabilistic process, and infers backwards from the actual words, to a distribution of topics. The topics can be discovered by this algorithm, and need not be specified in advance.

The mixed-initiative aspect is that, we ask people to describe each theme from the word cluster produced by LDA, then verify that it agrees reasonably well with a human interpretation of the story. This only needs to be done for each topic once a corpus is analyzed, not each time we interactively analyze a story. Figure 3 shows some of about 30 topics from a corpus of about 10,000 stories. 


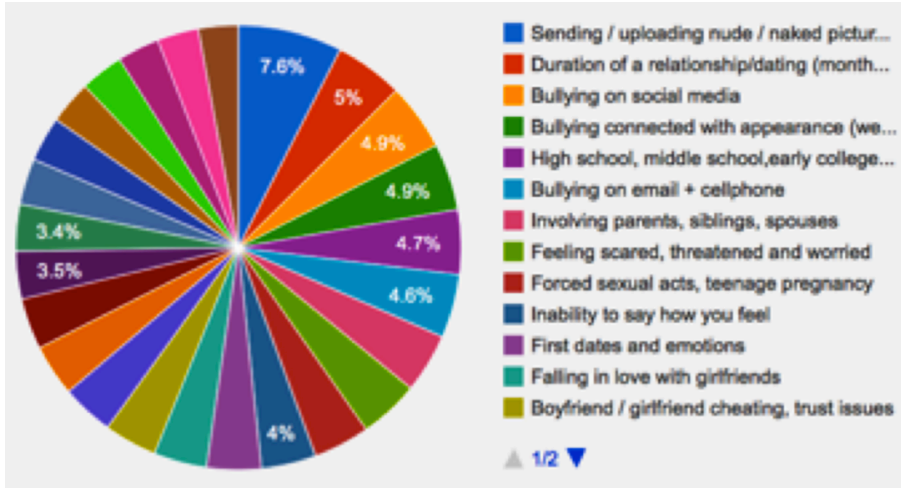

Figure 3. Theme distribution in the story corpus. 30 major themes were identified from 10,000 stories.

We also use a commonsense knowledge base [13] that contains over 1 million simple assertions about everyday life. For example, it might contain the assertion, "french fries make you fat", which helps us relate a story about eating too many french fries to the topic of insulting someone's appearance, a common bullying topic. We also augment the commonsense knowledge base with a more specialized knowledge base, BullySpace, containing specific knowledge about bullying topics, like racial and ethnic slurs.

In addition, we are also exploring finer grained methods of understanding the stories. While our current techniques relate to the topic of the entire story, we are also exploring modeling specific actors and events in the story, in order to recognize stereotypical story patterns like Schank's "scripts" [11]or Winston's Genesis system [15]. A companion paper [6] reports on some preliminary work.

\section{Interaction with "Over the Line"}

Figure 4 shows the screen with the system's response for finding stories similar to a given story. At top, the original story input by the user. Directly below it, a similar story retrieved by the system. The user can page through all matching stories, in order of relevance. The system also asks the user to rate the match (as well as rating the individual stories).

The "Resources" bar at the bottom is also personalized, using the computed story theme to filter the list of resources, taken from the other categories of information on the site. For example, if the inferred theme is, "pressure for having sex", the resource Dating Abuse Hot Line will be offered.

\section{Related Work}

Given the importance and ubiquity of this problem, it is surprising that there has been almost no prior work directly in the area of designing social network software to prevent and mitigate cyberbullying. There has been some work in educational games that simulate potentially bullying situations, e.g. [7], but these are fictional situations that do not directly relate to users' real-world lives.

\section{Evaluation and Future Work}

We conducted a preliminary user study with 9 participants, 5 male and 4 female, ranging in age from 18 to 22 . The age range is somewhat high relative to the ages at which peak rates of bullying occur, but several participants chose to present stories that occurred several years prior to the experiment, and commented that it would have been more effective for them at the younger age at which the incident 
occurred. Participants were shown the site as it is available to the public, and asked to relate a personal experience that they, or someone they knew, had experienced.

The experiment was primarily concerned with answering two questions: First, was the system able to find stories that matched well enough to be considered interesting and relevant to them? (Overall precision and recall of the match was not a goal for us.) Second, did they find the process of reading matched stories helpful in terms of feeling less alone, more able to reflect on their experience, or receiving useful advice?

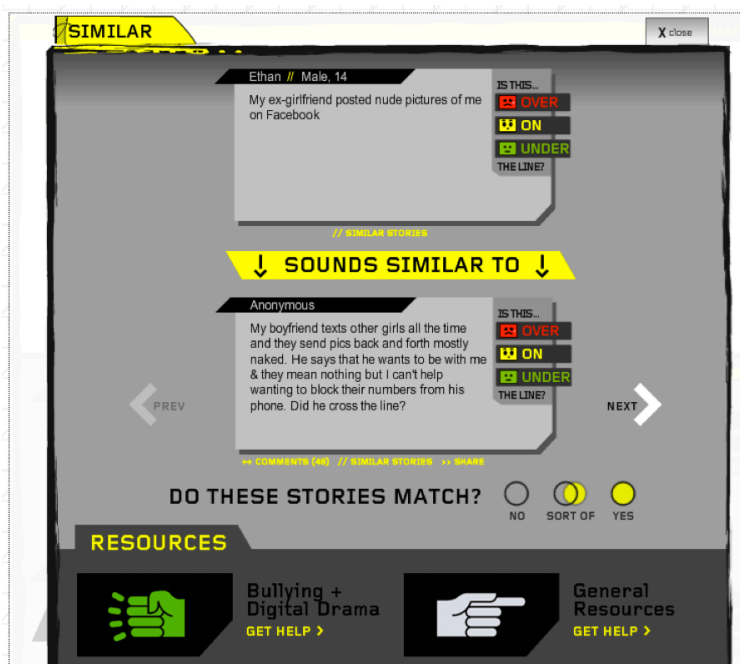

Figure 4 . The story matching screen.

5 of 9 participants ( $55 \%$ ) found at least some interesting and relevant matches among the (typically four or five) stories they examined. 2 participants $(22 \%)$ had mixed or doubtful results, and 2 (22\%) were unable to find any stories at all that satisfied them. All who received good matches found the system helpful, and one also found it helpful even though they did not rate the match well $(66 \%)$. Overall, we found these results quite encouraging. Future work will involve a control group receiving randomized stories, and perhaps a younger set of participants.

We will continue to experiment with better ways of matching stories, interactive improvement of the knowledge base and machine learning techniques. We would also like to generate personalized advice dynamically by taking advice written for a genera audience around one of the recognized story themes, and substituting in particulars of the user's story.

We are also exploring the potential for applications of these techniques to problems other than cyberbullying, such as support for autistic spectrum individuals.

\section{Acknowledgements}

We would like to thank Jason Rzepka of MTV for bringing our work to the public via their Over the Line site. We would like to thank Aneesh Chopra, former White House CTO, for sparking our collaboration at the 2011 White House Conference on Bullying Prevention. We thank DJ Ronde for conducting the experiments.

We wish to acknowledge the financial support of the Simons Center for the Social Brain at MIT, and Formspring, as well as the over 70 corporate and organizational sponsors of the MIT Media Lab. 


\section{References}

[1] Bazelon, Emily, Sticks and Stones: Defeating the Culture of Bullying, Random House, 2013.

[2] Blei, D., Ng, A., Jordan, M., Latent Dirichlet Allocation, Journal of Machine Learning Research, Vol 3, 993-1022, 2003.

[3] Dinakar, K., Jones, B., Havasi, C., Lieberman, H., Picard, R., Commonsense Reasoning for Detection, Prevention and Mitigation of Cyberbullying, ACM Transactions on Intelligent Interactive Systems (ACM TiiS), Volume 2, Number 3, 2012.

[4] Dinakar, K., Jones, B., Lieberman, H., Picard, R., Rosé, C. Thoman, M., Reichart, R., You Too?! MixedInitiative LDA Story Matching to Help Teens in Distress, International Conference on Weblogs and Social Media (ICWSM-12), Dublin, Ireland, June 2012.

[5] Jones, B., Reflective Interfaces: Assisting Teens with Stressful Situations Online, MS Thesis, MIT Media Lab, 2012

[6] Macbeth, J., Adeyama, H., Lieberman, H., Fry, C., Script-Based Story Matching for Cyberbullying Prevention, CHI Works in Progress, 2013.

[7] Mancilla-Caceres, J.F., Pu, W., Amir, E., and D. Espelage. Identifying Bullies with a Computer Game. In
Proceedings of the 26th AAAI Conference on Artificial Intelligence (AAAI-12), 2012.

[8] MTV Networks and Associated Press. (2011). MTVAP Digital Abuse Survey. Retrieved from http://www.athinline.org/pdfs/2011-MTVAP_Digital_Abuse_Study_Full.pdf

[9] MTV Networks, private communication.

[10] Olweus D. Bullying at School: What We Know and What We Can Do. Oxford, England: Blackwell; 1993.

[11] Schank, R., Abelson, R., Scripts, Plans, Goals and Understanding, Lawrence Erlbaum, 1977.

[12] Schön, D. (1983) The Reflective Practitioner, How Professionals Think In Action, Basic Books.

[13] Speer, R., Havasi, C., Lieberman, H., AnalogySpace: Reducing the Dimensionality of Commonsense Knowledge, Conference of the Association for the Advancement of Artificial Intelligence (AAAI-08), Chicago, July 2008.

[14] US Dept. of Education,

http://www.stopbullying.gov

[15] Winston, P., The Strong Story Hypothesis and the Directed Perception Hypothesis, AAAI Fall Symposium on Advances in Cognitive Systems, 2011. 